\title{
A Community-Centered Design Framework for Robot-Assisted Feeding Systems
}

\author{
Tapomayukh Bhattacharjee \\ Paul G. Allen School of CSE, \\ University of Washington, \\ Seattle, USA \\ tapo@cs.washington.edu
}

\author{
Maria E. Cabrera \\ Paul G. Allen School of CSE, \\ University of Washington, \\ Seattle, USA \\ mecu@cs.washington.edu
}

\author{
Anat Caspi \\ Paul G. Allen School of CSE, \\ University of Washington, \\ Seattle, USA \\ caspian@cs.washington.edu
}

\author{
Maya Cakmak \\ Paul G. Allen School of CSE, \\ University of Washington, \\ Seattle, USA \\ mcakmak@cs.washington.edu
}

\author{
Siddhartha S. Srinivasa \\ Paul G. Allen School of CSE, \\ University of Washington, \\ Seattle, USA \\ siddh@cs.washington.edu
}

\begin{abstract}
Robot-assisted feeding (RAF) systems offer enormous potential benefits to community-centered care-giving environments. However, developers of RAF technologies often focus on evaluating their standard transactional functionality, omitting the impact of such technologies in contexts that extend past the interaction of the robot and food receiver. RAF technologies have complex social, cultural and self-identity implications, since a "meal" extends well beyond the simple provisioning of nourishment. To better understand these implications we conducted a contextual inquiry in an assisted-living community with five potential care recipients and five caregivers, as well as interviews with fifteen domain experts including occupational therapists and feeding specialists. Based on our findings from these studies, we developed a new framework for RAF technologies that formulates this vital task as a communitycentered relational service. We then use this framework to qualitatively and quantitatively assess three existing feeding systems and identify areas of improvement. Our work reveals new insights about stakeholders of RAF technologies and provides a roadmap for technology developers to better serve the needs of these stakeholders.
\end{abstract}

\section{CCS Concepts}

-Human-centered computing $\rightarrow$ Empirical studies in accessibility; $\cdot$ Social and professional topics $\rightarrow$ Assistive technologies; People with disabilities; •Computer systems organization $\rightarrow$ Robotic autonomy;

Permission to make digital or hard copies of all or part of this work for personal or classroom use is granted without fee provided that copies are not made or distributed for profit or commercial advantage and that copies bear this notice and the full citation on the first page. Copyrights for components of this work owned by others than ACM must be honored. Abstracting with credit is permitted. To copy otherwise, or republish, to post on servers or to redistribute to lists, requires prior specific permission and/or a fee. Request permissions from Permissions@acm.org.

ASSETS '19, October 28-30, 2019, Pittsburgh, PA, USA

Copyright (C) 2019 Association of Computing Machinery.

ACM ISBN 978-1-4503-6676-2/19/10 ...\$15.00.

http://dx.doi.org/10.1145/3308561.3353803

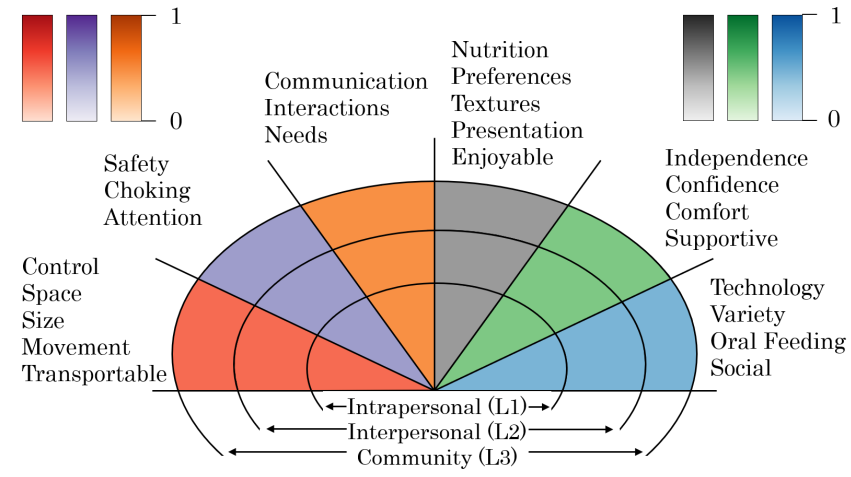

Figure 1: Multi-level Contextual Framework

Author Keywords

Assistive Feeding; Assistive Robotics.

\section{INTRODUCTION}

Studies have consistently shown that long-term care recipients who live in the community have far better clinical outcomes than those in institutionalized settings [51]. Nearly $18.7 \%$ percent of the non-institutionalized US population live with a disability [21]. Of these, about 12.3 million need assistance with one or more activities of daily living (ADLs), such as feeding, bathing, or dressing. Indeed, needing help with one or more ADLs is the most cited reason for moving to assisted or institutionalized living $[54,1]$. Moreover, depression and disability can worsen after ADL assistance is initiated, perhaps due to loss of regular routines that could worsen disability [46]. Sources of support for people with impaired levels of functional capacity in the community are typically family members, which make up an estimated $20 \%$ of the adult population in the US [34]. The care network increases in both size and scope as the care recipient's functional capacity declines. Hence, ADL assistance and management concerns many more individuals and communities beyond the person who requires functional support. 
Major efforts in the personal robotics community have created robotic solutions to increase independence or prolong independence in self-feeding [22]. Robot-assisted feeding (RAF) in itself poses multifaceted technical challenges. To date, most approaches to defining the RAF design space have focused on technology function and the care recipient, addressing specific sub-tasks in the food acquisition and transfer chain. These RAF systems were considered functional as long as they could pick up a food item and transfer it to a care-recipient. However, assistive robots do not exist in isolation, nor as an isolated unit with their care-recipient. There is an opportunity to design assistive self-care robot technologies that satisfy not only the functional requirements of performing an ADL, but also serve more of a supportive relational role.

We emphasize that this shift from transactional to relational service technology is aligned with the recent movement in human-computer interaction toward increasing the accessibility of technology using social models of disability (e.g., [35, 50]). We aim to understand how personal robotic solutions can be contextually appropriate in an assisted self-care setting, particularly in the case of ADLs, and be seamlessly integrated into a complex home environment. That is, how we can design robot-assisted self-care technology to support the needs of all participants in the home environment and scaffold interactions that promote agency for the robot-assisted individual while also increasing support for other individuals in the support network. We believe that a shift is needed in the assistive robotics for ADL community. This shift would be the first step in community-led ADL management. This, in turn, will promote wider adoption of assistive robotics.

This work offers three key contributions:

- A novel adaptation of a multi-level model from socialecological theory to analyze RAF systems accounting for transactional, relational, community, and social aspects.

- A proposed systematic design framework for evaluating RAF systems informed by our multi-level contextual inquiry with stakeholders.

- Analyzing three state-of-the-art existing RAF systems using our evaluation framework to demonstrate how it can be instrumental in understanding technology design gaps and priorities.

\section{BACKGROUND}

Our main tenet is that carefully-constructed, communitycentered design frameworks to develop assistive technology can drive innovations in how we create robotic technologies that are embraced and adopted in homes and living environments. This can be used to improve the community living experience for individuals with disabilities and their caregivers. Frameworks of study surrounding the design process of public services within complex social contexts provide us with trialed analytical frameworks. Our study insights, for example, are consistent with frameworks of community living and family centered care; frameworks which have gained increasing attention as a core determinant of care quality since a seminal report by the Institute of Medicine [68].
We take feeding, key among ADLs, as an example. The functional requirement of feeding is to nourish the body with food, but care recipients often reject feeding assistance because of the intricately complex association that food and meals have with self identity, cultural values, family life, and emotional well being [66]. Hence, there are wide ranging factors beyond technical efficacy: relational, emotional, community, and social factors that interact, influence and are influenced by RAF technologies. Design and development of assistive technologies should be informed by these factors and address the physical, cognitive, emotional, and social gaps that remain, even when an individual is functionally assisted with ADLs.

The social-ecological model (SEM) $[28,55]$ provides a conceptual framework to deconstruct complex social contexts like feeding and systematize the analysis, design and evaluation of RAF systems. SEM conceptualizes the social world in four levels of influence. These are: (L1) individual or intrapersonal, (L2) interpersonal, and (L3) community or institutional, and (L4) societal (indicating macro-level societal, policy or regulatory influences). In this paper, we concentrate on the inner three levels (Fig. 1), where L1 involves the care-recipient and caregiver pair, L2 involves family members, and L3 involves the larger social effectors. RAF design decisions involving these spheres will have the greatest impact on adoption and acceptance of robotic applications in the community. We leave out the outermost level (L4), which involves interrogating processes and decision-making at the legislative or national level, as it is outside the scope of our research, but remains critical in adoption of RAF technologies.

To further structure the multi-level approach and focus its use in the context of community-supporting-technology, we inform our design framework with contextual dimensions currently used in evaluating robot-assisted transportation applications at the USDOT [26]. The six contextual dimensions (described below) are helpful in selecting and applying appropriate performance indicators and measures that are integral to community-centered RAF design decisions. Our workflow (described in section 6) analyzes RAF systems per these contexts along the multiple social levels offered by the SEM. The result is a workflow for comparative evaluations for RAF systems in a community-centered approach.

To the best of our knowledge, there are no groups currently framing assistive robotic technologies as a social service intervention. The approach in itself provides broader and layered contexts of analysis, extending beyond previous framings that focus on examining the interaction between the technology and its immediate user. Specifically, the framework provides tools to understand the influence by and on related users in broadening context-layers (care recipients, caregivers, home care personnel, therapists, care providers, social workers, etc). The utility of defining such a framework is demonstrated by using it to differentiate among different RAF solutions. The framework is used to compare several existing RAFs, enabling us, in the process, to identify key properties of the technology and the design space. The comparison highlights areas where existing RAFs provide extensive support and those in which they are deficient, suggesting a future research agenda. 

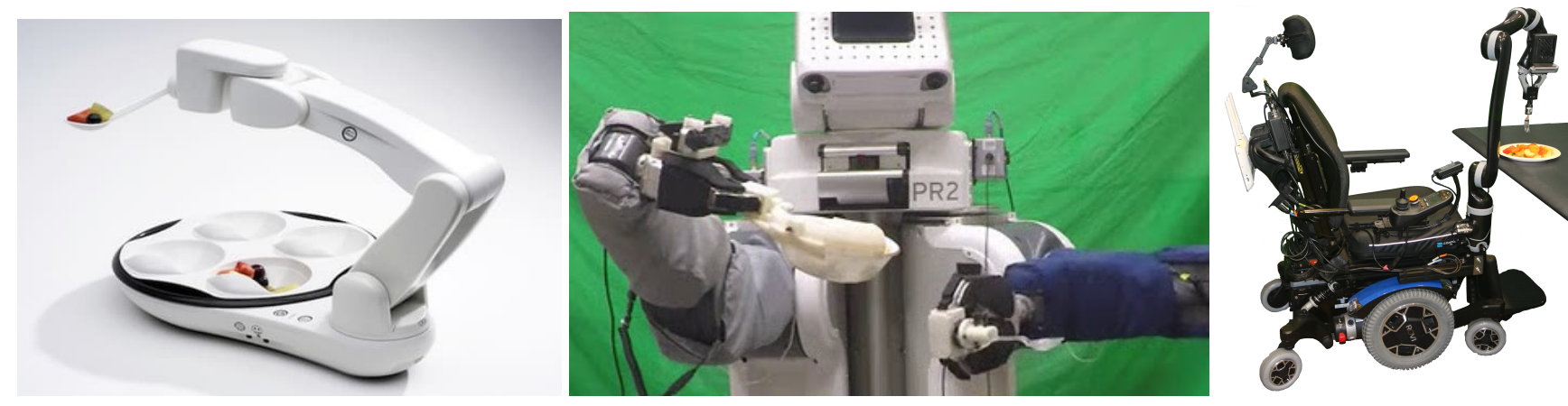

Figure 2: Three RAF systems assessed within the developed framework: (Left) Obi [7], (Middle) PR2 [62], (Right) ADA [36].

\section{RELATED WORK}

\section{Robot-Assisted Feeding systems}

Several specialized feeding devices for people with disabilities have been developed in the past decade, three of which are shown in Fig. 2. Although there are several commercially available RAF systems in distribution $[7,6,4,3,9,11,5,14$, 10] that use a robotic arm to scoop food with a spoon, they have lacked widespread acceptance. There are also RAF systems that were developed in research labs, either as table-mounted robotic arms $[25,71,69,59,65,64,41,43,47,75,85,44,78$, $77,87,86,74]$, general purpose mobile manipulators $[18,67$, 62], or wheelchair-mounted robotic arms [19, 16, 31, 42, 49, $32,36]$.

A comprehensive review of meal assistance robots is given in $[57,76,45,23]$. Some assistive systems focus less on full autonomy and more on support or adaptation to the user. For example, several systems emphasize tremor cancellation during feeding task [58, 13,63], while others provide arm support for users who can move their arm but do not have sufficient muscular strength for continued large movements [80, 12, 15]. Park et al. [62] developed a semi-solid food acquisition system for assistive feeding using a general purpose manipulator to scoop yogurt with a spoon. They also developed an anomaly detection framework for assistive feeding using multiple sensing modalities [60, 61]. Herlant [40] developed a robotic system that uses a fork to skewer solid food items. Gallenberger et al. [36] can detect a solid food item specified by a user and pick it up using a fork autonomously. Canal et al. [24] developed an intelligent personalization framework for RAF systems that can adapt to user preferences. McColl and Nejat [53] performed a study with an expressive socially assistive robot Brian 2.1 and older adults during meal time in a care facility and explored ways in which a socially assistive robot can provide cognitive assistance during the feeding activity to analyze user engagement and compliance during meal time.

\section{Contextual inquiries in related assistive technologies}

Researchers have also conducted contextual inquiries to analyze other assistive technologies, as well as their opportunities and challenges. Szpiro et al. [73] conducted a contextual inquiry to understand the opportunities and challenges of using mainstream computing devices by people with low vision. Naftali and Findlater [56] performed a contextual session to analyze how users with motor impairments use smartphones in their daily lives. Maciuszek et al. [48] constructed a functional design space of electronic assistive technologies by conducting a study with both the users and designers of the technology. Overall, in the literature, researchers have conducted studies with multiple stakeholders of varied assistive technologies to analyze their functions and scope of usage.

\section{A STUDY WITH STAKEHOLDERS}

The value of this paper lies in connecting systems thinking and assistive robotic design practices while attempting to improve adoption of robots in everyday living. Our design framework proposes a shift towards designing enabling environments that support multiple actors at multiple layers of the social network surrounding a care recipient, and away from a myopic focus on a single user and a fully-automated method to replace human caregivers. The goal of our contextual user study was to better map and understand how social relationships may shape and inform new supportive design objectives and tasks for the RAF system when re-conceptualized as a technology supporting the entire network. We consider this a community-led relational approach. To improve our understanding of the sociology surrounding feeding, we performed a contextual inquiry with experts at various layers: domain experts (occupational therapists, therapeutic recreational specialists and feeding specialists) as well as local needs experts (care recipients and caregivers).

\section{Multi-Level Contextual Inquiry}

The goal of our contextual inquiry was to provide insight into all potential stakeholders in the social service the robots may provide. We used a combination of surveys and contextual inquiry to capture a mix of quantitative and qualitative data describing stakeholders' background, preferred methods of feeding (if applicable), attitudes, practices, relationships and information needs, concerning feeding. Our study included 15 experts, and 5 care-recipients with 5 of their caregivers. We describe our methods more specifically below.

\section{Contextual Inquiry in a Community Setting}

Care-recipients and caregivers were engaged in a communal meal in their home setting where researchers observed the meal activity. Care-recipients were assisted in a typical fashion by the participant's typical caregiver. The user participants were 2 female and 3 males, all wheelchair users with neck 
motion ability and showed no evident swallowing problems. The caregivers were 3 female and 2 males, registered nurses and nurse practitioners among their occupations.

This contextual inquiry had three main components:

1. Semi-Structured Interview: Understanding the nuances of the feeding task and its challenges

2. Activity: Collecting observations while having a meal together

3. Introduce idea: Showing a video of our assistive feeding robot and getting their impressions and feedback

We conducted the study by asking participants the questions verbally and then recording responses. One care-recipient had speech impairments and used a tablet to respond to the questions, while others responded verbally. During the study, we used multiple go-pro cameras to record the video and audio which were transcribed later for qualitative analyses.

\section{Inquiry with Experts}

We developed an expert survey to gather information from participants who are very familiar with feeding in care settings, and who may be able to influence (to varying degrees) feeding assistance in the community setting. Fifteen experts responded using an electronic questionnaire. The group included occupational therapists (OT), therapeutic recreation specialists, physical therapists, assistive technology professionals, speech-language pathologists (SLP), surgical technologists, and executive director of a non-profit organization focused on independence and technology. Out of the 15 experts, 13 were female, the age ranges from 23 to 63 years of age, with a mean age of $43 \pm 12$ years. Regarding the experience of feeding someone with mobility impairments, $86.7 \%$ of the surveyed experts responded affirmatively. Years of professional experience ranged from 3 to 40 years in their particular occupation, with a mean of $18 \pm 12$ years. One of our surveyed experts happens to have mobility impairments, so he was surveyed as both an expert and a user. The electronic survey for experts contained twenty open-ended questions as well as twelve Likert-scale questions to gather their impressions and concerns involving a robot for assistance with feeding.

\section{STUDY FINDINGS}

In this section, we present the findings of the multi-level contextual inquiry with users, caregivers and experts, as well as our observations during the feeding activity. To organize the collected data, we segregate our analysis by the spheres of influence suggested by the social-ecological model as they would be applied to conducting and evaluating RAF systems. Within each sphere, the participant responses are further interrogated using six contexts of evaluation [26]: technical function, technological robustness, information gaps, usability, user empowerment and social acceptance, and system integration. These contexts provide multiple perspectives to help in mapping the information respondents told us to technology design indicators that would impact the implementation and development of a RAF system. Our analysis of the collected data using the framework as described above, leads us to identifying (within each context of evaluation) indicators that can be used to evaluate RAF system impact in each sphere. Indicators are the constructs, activities, and behaviors that the RAF technology would operationalize to effect change at the particular sphere. Measures are the specific tools that can be used to evaluate the change. The indicators under each of these evaluation contexts were qualitatively obtained using the stakeholder interviews. Our interviews with stakeholders were analyzed independently by two authors, coded using identified keywords, and then discussed to reach a $100 \%$ inter-rater reliability score. In this manner, the community's data derives the evaluation framework that is used in later sections to compare existing RAF systems.

\section{Technical Function:}

This evaluation context involves system aspects that directly impact functional metrics related to feeding. Considering the wide range of user capabilities and dietary needs, a RAF system needs to handle multiple tools necessary to manipulate different food types and textures. From our surveyed users, food choices ranged from pre-cut pieces of meats to soft foods such as mashed potatoes and pieces of fruits. Experts such as E6 responded to the nutritional challenges with: "ensuring adequate caloric intake across a variety of food textures and types that are safe for the patient". As we move across the different levels of influence food preference may change from a few items or food types at the individual level, while interpersonal and community levels may require the RAF system to handle greater variety.

Caregivers noted the feeding task is physically challenging. This also falls under the context of technical function, as the RAF system could help resolve some of the ergonomic difficulties associated with the task. During our visit, we noticed that the caregivers stood next to the care-recipient holding the bowls in their hands and had to bend or adjust to feed each care-recipient (see Fig. 3). Expert responses also indicated that proper utensil positioning in proximity to mouth could both improve feeding efficiency and achieving proper nutrition, but also maximize care recipient's independence and participation in the feeding process. One of the surveyed caregivers, C5, described her motions during feeding as: "careful smooth and level motions with a spoon,(...) to ensure I have a good grasp and won't drop the food prior to releasing it into the patient's mouth". The surveyed caregivers did not mention ergonomics explicitly, but $\mathrm{C} 1$ did comment that the robot needs to "show flexibility" in motion and grasping, perhaps considering her own variety of postures and motions during feeding. These considerations also change as we move between levels, since the system's size and footprint might not be as important in the care recipient's home, whereas positioning the RAF system at a restaurant may be challenging or prohibitive. Several experts also addressed the system's physical qualities. They mentioned the robot size should be small (sleek, compact), it should be quiet, it should not draw unnecessary attention. Other experts mentioned the importance of offering a "guideline for how much space is needed around the client and the robot so the arm doesn't end up bumping into anyone". Safety is of paramount importance. S3 and S4 mentioned an emergency stop button as an important feature for the system. These are relevant in all three spheres. 

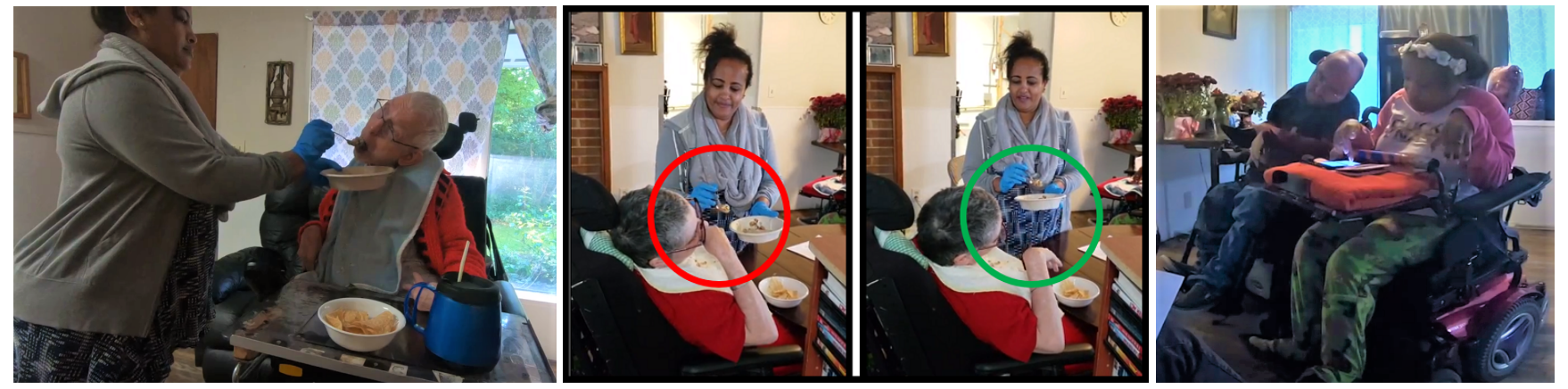

Figure 3: Observations from caregiver-assisted feeding activity during our contextual inquiry: (Left) Caregiver feeding a care-recipient, (Middle) Care-recipient communicates intent, (Right) Different user interface.

\section{Technology Robustness:}

This context addresses how the RAF system would improve safety and reliability by addressing anomalies, uncertainty, and risk. Our participants noted RAF system must adapt to changing environment. At the intrapersonal sphere, a RAF system must adapt to care-recipient positional changes. At the interpersonal level, it must avoid collisions with people or objects within its working space. One of the surveyed caregivers also mentioned robustness in terms of the system maintaining functionality through rough handling and falls. An expert (E3) also commented on robustness regarding the "possibility of (the robot) breaking or needing repairs that could leave individuals stranded if dependent on the robot". Being able to detect these failures and correct them are important indicators in this context. Projecting this indicator to the community sphere, functionality must be robust to environmental complexity (e.g., lighting conditions and background noise). Experts also added comments related to food-intake safety. For example, detecting if a care-recipient may be choking and notifying someone, providing reminders to drink during the meal or to slow down food intake. privacy and security of user-data, relative to data collected or generated by the RAF would be additional viable indicators under this context.

\section{Information Gaps:}

Care recipients or caregivers may have functional or situational sensory or cognitive limitations that create gaps in the system's communication with users. The system should provide multiple formats for feedback, and multiple modalities for executing system functions. Even when experts generally suggested voice commands if there are mobility issues, they also acknowledge that some care-recipients may need other methods such as gestures, head or shoulder movements, gaze, or adapt the system's interactions to available AAC (Augmentative and Alternative Communication) technology, or even texting using a tablet as was the case for one of our participants with speech impairment (see Fig. 3). At the interpersonal sphere, potentially less structured environments may require redundant formats, for example, between verbal commands and button pushes. Analogously, if the RAF system includes vocal output interactions, those might become bothersome to third parties at the interpersonal sphere.

\section{Usability:}

In the usability context, the RAF addresses indicators that impact customization and user preferential aspects. At the intrapersonal sphere, most participants discussed necessary adaptations to care-recipient preferences and needs. Customization capabilities to adapt to user preference is a fundamental indicator within the usability context. Caregivers and experts highlighted the importance of bite size tuning. Seven experts mentioned 'bite size' as an important consideration while feeding someone. S1, S3, and S4 mentioned that food needs to be cut, while S5 prefers to have the food cut as he eats. S3 said that she needs smaller pieces "to avoid difficulties chewing/swallowing". The importance of honoring the preferences of a user was also mentioned by caregivers. We highlight C1's response in particular: "we notice he wants his mouth to be full".

Regarding timing for transferring the bite, the surveyed caregivers agreed that they detect care recipient gestures (open their mouths or nod head), or wait for a positive response after querying if they are ready for another bite. This translates to two indicators that are dependent on properly detecting whether mouth is open or not. When considering the interpersonal or community level, determining when to transfer the bite might become more dynamic to account for interactions such as participating in a conversation. Also, the mouth detecting feature needs to be more robust to detect only the face and mouth of the care recipient in an environment with multiple people.

Additionally, eight experts mentioned 'pacing' as an important consideration while feeding someone. S2 says that it should be up to the user to select the speed of the robot when it is feeding him. To highlight this point, S3 thinks that using a robot feeding would be faster, while S4 feels sometimes the caregivers hurry and expects "... the robot would adjust more to our time". Experts such as E9 described the assisted feeding activity as "time consuming, you can't really do something else at the same time", whereas E12 addresses the challenges with more empathetic perspective: "having patience to allow natural eating pace". This translates to the robotic feeding system allowing the caregiver some flexibility to focus their attention on other tasks or activities, which may include companionship and social interactions with the care recipient. Bite sequence is also an important consideration. S3 and S4 mentioned that 
sometimes they choose what they want in their next bite, and sometimes they do not. This issue also relates to how much each user can participate during the meal, maximizing their independence and making the activity a positive experience, and "more than a nutritional exchange" (E2). Additional usability indicators (important in all assistive robotic systems) impact learnability, memorability (ease of training and retaining training on use of the technology). Usability also prevents potential user error. At the institutional level, one indicator could be the ability to identify patient trends (such as tracking decline in function or skill).

\section{User Empowerment and Social Acceptance:}

There are additional emotional and social supports that the RAF system can facilitate associated with eating as a daily activity that are addressed through this context. Several experts addressed the user's comfort level with phrases such as "preserve a patient's dignity" (E12), "person being fed may or may not enjoy having a person feeding them" (E6), and sometimes the user may "prefer a family member feed (them) because is more enjoyable" (E13). E3 commented:

Oftentimes meals are a time for socialization and eating. Often for people with disabilities that socialization piece is taken away because the caregiver may be so focused on feeding and not facilitate that social piece or they may be in a rush to finish feeding.

From the users' perspective, S3 and S4 mentioned feeling "embarrassed" when asking someone else to help them eat. S2 takes it further by imagining a social interaction where he meets someone in a coffee-house and the robot assists him with eating a donut. One of the caregivers (C3) also mentions the potential for increasing the care-recipient's self-esteem. On the topic of independence, the surveyed experts and caregivers vastly agree on that aspect being one of the greatest potential benefits of adopting a robotic feeding system. S5 also mentioned feeling like a child when he is being fed. This was also addressed by an expert (E9): "a degree of infantilization that can occur. It's such a personal thing to have someone shoveling food in your mouth, really represents a loss of independence across the board". Some experts also mention that the presence of an interaction-less robotic feeding system may result in potential social isolation. C2 explained that when a caregiver feeds a care recipient there are interactions that would go missing with a robotic system: "when we feed, we talk, we laugh, we ask how they are doing!". Experts further expand this issue by assigning the caregivers a role of companionship. E2 said:

When self feeding is possible in older patients, then the eating process can shift to one where the caregiver is present as a social partner/companion around a meal rather than having the experience be one that feels tied to a dependent relationship

As a part of the experts' survey, several Likert-scale questions addressed concerns or potential benefits of an assistive robotic system:

1. I am delighted to think of the INDEPENDENCE assistive robots can add to the life of a person with a disability.
2. I am delighted to think of the POSSIBLE TASKS assistive robots can perform in the life of a person with a disability.

3. I am concerned that individuals with disabilities would feel UNEASY if they had to use the help of robots to eat.

4. I am concerned that caregivers would feel UNEASY if they were given a job where they had to use robots.

5. I am concerned that robots would be a BAD INFLUENCE in the life of a caregiver.

6. I am concerned that robots would be a BAD ADDITION in the life of a person with a disability.

7. I feel it is possible for individuals with disabilities to DEPEND TOO MUCH on robots.

The results are presented in Fig. 4. Overall, experts strongly agreed with the idea of increased independence and greater number of tasks that a person with a disability could accomplish using a robotic feeding system. Regarding the concerns of including robots in the process of feeding, experts were not concerned with the inclusion of technology for either stakeholder, care-recipient or caregiver. Experts also disagreed to consider adding a robotic system as a bad influence to caregivers or a bad addition to the care-recipients' lives. We averaged the Likert-scale responses across experts and used a t-test to compare them against a neutral statement, also used as null hypothesis (Likert score $=3$ ). Normality assumption was checked using Skewness and Kurtosis. Using the experimental data, we found statistical significance for all the questions except (4) and (5) with $p<0.05$.

\section{System Integration:}

This context encompasses how the robotic system can be integrated with other systems in day to day life. For example, the robotic system would need to share power resources with the electric chair. This leads to managing resources and maybe incorporating low-battery warnings and integrating with other assistive devices and alarm systems (for safety) to notify caregivers when anomalies are detected. Using this perspective, it is important to consider the impact when dealing with different levels of influence. To use the battery level as an example, in an intrapersonal level battery life might not even be considered while feeding since charging could be done at the same time. This may not be the case in the community level, where both wheelchair and RAF system share the battery resource and a minimum battery level might be required to consider having a meal in a restaurant. From our observations during the feeding activities, S5 was already using multiple assistive devices and technologies based on sip and puff.

\section{A WORKFLOW FOR EVALUATION}

We developed the following workflow for testing RAF technologies based on goal-driven contexts. First, any assistive technology needs to define the problem. In our case, the problem is to develop a robotic system that can assist people with feeding as a long-term care solution. The next step is identifying stakeholders. Based on the problem and the stakeholders, the system needs to be developed and evaluated. 


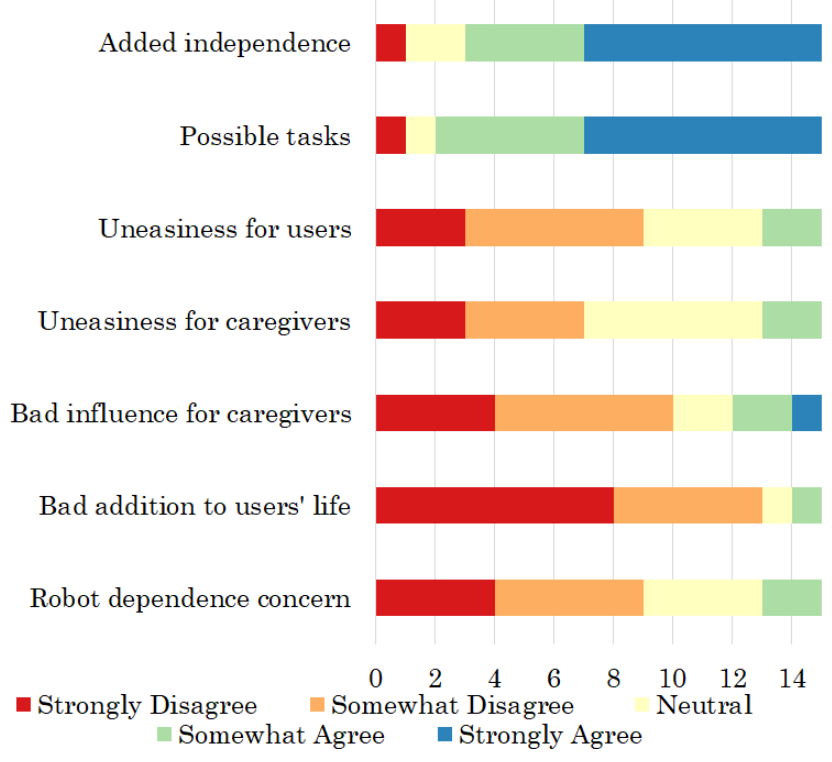

Figure 4: Experts' responses to Likert-scaled questions.

\section{Identifying Stakeholders}

This includes understanding different demographic factors that this technology attempts to address, including breakdowns by axes considering mobility profile (as defined in the framework), gender, race, environment, location, socioeconomic status and educational level. Usual stakeholders for RAF are people with upper-mobility impairments with conditions such as cerebrovascular diseases like strokes [79], Parkinson's, arthritis, multiple sclerosis [17], spinal cord injuries [52], bilateral amputations, and many others which can render individuals unable to eat on their own accord.

\section{Evaluation Indicators}

The goal for a RAF system is to increase the care-recipient's independence and maximize their participation during the activity while still providing adequate nutrition. Based on the problem definition and the stakeholders, the evaluation criteria can be specified from among the indicators given below. These indicators hinge on our observations during the feeding activity of the contextual inquiry as well as feedback from care-recipients, caregivers, and experts. We have come up with the following evaluation indicators:

\section{Technical Function.}

The evaluation of this context involves performance indicators related to bite acquisition and transfer efficiency as well as safety.

- Range offood items: This tells us what kind of food items can the RAF system successfully feed a person.

- Bite acquisition success rate: This tells us how successful a bite acquisition attempt by a robot is, for a variety of food items.

- Bite transfer success rate: This tells us how successful a bite transfer attempt by a robot is, for a variety of food items.
- Face detection: This is important for the robot to be able to detect where the mouth is.

- User interface: This tells us if a user interface is available for a robotic system.

- Reachability and Mobility:The robotic system should have a big enough workspace to be able to reach and pick food up from a range of plate positions and should be mobile such that the user can eat food wherever they want to, in their own homes, with their family, or even in a restaurant.

- Collision Detection:Safety is of paramount importance. The robotic system should be able to detect collisions and stop immediately in case of unexpected collisions.

- E-stop: The robotic system should have multiple redundant features such as emergency-stops and/or other modalities such as speech to command the robot to stop whenever they want.

\section{Technology Robustness.}

The evaluation related to this context mainly addresses the capability of a RAF system to be robust to perturbations.

- Collision Avoidance: Ability to adapt its trajectory to avoid colliding with unexpected static or dynamic objects or body parts in its path.

- Visual Servoing: Ability to adapt its motion to the movements of the care-recipient's neck/head or movements of the plate or bowl during the course of a meal.

- Anomaly detection: Ability to detect anomalies whether it is task, software, hardware, or environment-based.

- Anomaly correction: Ability to correct task-based anomalies such as bite-acquisition and bite-transfer failures.

Information Gaps.

The evaluation related to this context mainly addresses the robot-user communication of information.

- User interface suitability: Depending on the target subpopulation, a robotic system needs to be equipped with a user interface with a suitable modality that can be used to communicate.

- Robot communication interface: It is equally important for a robotic system to communicate what it is doing at a particular feeding phase so that care-recipient feels more connected to it. This could be especially important for the blind community.

Usability.

The evaluation related to this context focuses on the customization and usability aspects based on inputs from our stakeholders.

- Ease of bite transfer: This tells us how easy a bite transfer attempt for a robotic system is.

- Bite transfer fluency: One of the factors of a fluent bite transfer, which requires fluent human-robot interaction, is proper bite timing. Proper bite timing is important for an 
enjoyable eating experience so the care-recipient does not feel rushed or is kept waiting.

- Bite size adaptability: Ability to adapt how much food it picks up in one bite cycle according to a care-recipient's preference.

- Bite timing adaptability: Ability to adapt when to feed a care-recipient based on their preference so that the entire meal is a positive experience for the care-recipient.

- Bite sequence adaptability: Ability to feed food items in certain order based on a care-recipient's preference or their nutrition requirements to make the entire meal an enjoyable experience.

\section{User Empowerment and Social Acceptance.}

The evaluation related to this context addresses user empowerment and how likely it is for them to socially accept this technology in longer term. We base these indicators on Technology Acceptance Models (TAMs) [29, 38, 33, 30, 82, 83, 84, 27, 70, 81, 39, 72].

- Perceived Usefulness: Be useful such that the care recipient's subjective probability using the technology will increase his or her performance

- Perceived Ease of Use: Degree to which the care-recipient expects that using the robotic system would be free of effort for the feeding task.

- Attitude: A care-recipient's positive or negative feelings (evaluative affect) about using the robotic feeding system.

- Intention to Use: A care-recipient's intention to perform a specific behavior to use the robotic system for feeding tasks.

- Perceived Enjoyment: Extent to which the activity of using the robotic system is perceived to be enjoyable in its own right, apart from any performance consequences that may be anticipated.

\section{System Integration.}

The evaluation related to this context addresses the compatibility of the robotic system with other systems in the home so that proper integration is achieved.

- Onboard electronics: Onboard sensing, computation, and networking capabilities so that it can feed people with minimal changes to the in-situ environment of the carerecipient and can communicate with other networked devices if needed.

- Anomaly monitoring capability: Integration with an alarm system to indicate low levels of battery, choking hazards, or anything else that needs immediate attention such as irregular bib placement, irregular hydration etc.

- Integration with other assistive devices: Ability to integrate with other assistive devices that are present in a carerecipient's home such as a wheelchair or an autobed [37].

\section{EVALUATING ROBOT-ASSISTED FEEDING SYSTEMS}

In this section, we describe some of the existing robotic feeding systems and evaluate them according to our framework. We categorize these assistive robotic feeding systems into three categories: 1) table-mounted robotic arms, 2) general purpose mobile manipulators, and 3) wheelchair-mounted robotic arms and analyze one representative robot from each of these categories.

\section{Comparing existing robot-assisted feeding systems}

\section{Table-mounted Robotic Arms: Obi}

Out of the myriads of table-mounted robotic arms for feeding mentioned in Section 3, here we take a closer look at Obi. Obi is a commercially available table-mounted portable assistive feeding device (See Fig. 2, left) and weighs approximately $7 \mathrm{lbs}$. It can detect collisions and can feed up to 4 hours with one charge. It has minimal autonomy but has a teaching mode by which its trajectories can be pre-programmed for later use. Obi has two user controls to command food delivery including multi-directional food capture, food re-positioning, and portion control, and to choose among four food bowls. It uses a washable spoon to scoop food from the food bowls.

\section{General Purpose Mobile Manipulators: PR2}

Out of the general purpose mobile manipulators for feeding mentioned in Section 3, here we focus on PR2. PR2 is a 32-DoF general-purpose mobile robot manipulator [20] with an omni-directional mobile base, a 1-DoF telescoping spine, and two 7-DoF back-drivable arms for developing a RAF system [62]. The robot holds a bowl and a utensil and the system can accept commands from a user via a graphical user interface. The system can scoop a spoonful of food by locating the highest food location in a bowl using a headmounted RGBD camera, feeds a user by estimating the face and mouth pose using a wrist-mounted camera, and can also wipe the spoon [62]. While running the tasks, the system runs a multimodal execution monitor to detect anomalous behaviors using 6 different sensors [61].

\section{Wheelchair-mounted Robotic Arms: ADA}

Out of the wheelchair-mounted robotic arms for feeding mentioned in Section 3, we focus on our autonomous robotic feeding system, ADA [36]. Our system consists of a 6-DoF JACO robotic arm [2] mounted on a powered ROVI wheelchair [8] to mimic similar setups used in real homes. The robotic arm has a wrist-mounted camera and has 2 fingers equipped with tactile sensors that grab a fork. We used tactile sensing to control the end effector forces during skewering and to detect if food acquisition was successful as well as if the fork hits something unexpectedly to improve safety. Depending on what food item a user selects, the robot perceives the food item on the plate using perception algorithms [36] and decides the best strategy to pick it up. Once the algorithms decide the strategy, the robot arm servoes to it using feedback from the visual modality, acquires the bite using feedback from the haptic modality [18], and then feeds it to a person by detecting the face and servoing to it using feedback from the visual modality. 
Table 1: Evaluation of three existing robot-assisted feeding systems: darker colors and larger circles indicate higher ratings

\begin{tabular}{|c|c|c|c|c|c|c|c|c|c|c|}
\hline \multirow{2}{*}{ Evaluation Contexts } & \multirow{2}{*}{ Indicators } & \multicolumn{3}{|c|}{ Obi } & \multicolumn{3}{|c|}{$\overline{\text { PR2 }}$} & \multicolumn{3}{|c|}{$\overline{\mathrm{ADA}}$} \\
\hline & & L1 & L2 & L3 & L1 & L2 & L3 & L1 & L2 & L3 \\
\hline \multirow{8}{*}{ Technical Function } & Range of food items ${ }^{\dagger}$ & 0 & O & O & $\bullet$ & - & - & 0 & $\bullet$ & ○ \\
\hline & Bite acquisition success rate & - & - & - & - & - & - & 0 & - & ○ \\
\hline & Bite transfer success rate & - & - & - & - & - & - & 0 & $\bullet$ & - \\
\hline & Face Detection & $x$ & $x$ & $x$ & & 0 & 0 & & 0 & 0 \\
\hline & User interface availability & O & O & O & & O & 0 & & & 0 \\
\hline & Reachability and mobility ${ }^{\otimes}$ & 0 & - & - & & 0 & - & & & \\
\hline & Collision detection & & 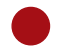 & & & 0 & 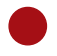 & & & \\
\hline & E-stop & & 0 & 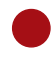 & & O & & & & \\
\hline \multirow{4}{*}{ Technology Robustness } & Collision avoidance & $x$ & $x$ & $x$ & O & $\circ$ & $\circ$ & & O & $\circ$ \\
\hline & Visual servoing & $x$ & $x$ & $x$ & $x$ & $x$ & $x$ & 0 & 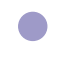 & - \\
\hline & Anomaly detection * & $x$ & $x$ & $x$ & O & O & 0 & 0 & & ○ \\
\hline & Anomaly correction ${ }^{*}$ & $x$ & $x$ & $x$ & O & O & O & - & - & - \\
\hline \multirow{2}{*}{ Information Gaps } & User interface suitability $^{\circ}$ & O & O & O & O & O & O & O & O & 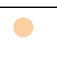 \\
\hline & Robot communication interface & $x$ & $x$ & $x$ & 0 & 0 & 0 & O & 0 & ○ \\
\hline \multirow{5}{*}{ Usability } & Ease of bite transfer & - & - & - & - & - & - & 0 & O & 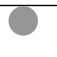 \\
\hline & Bite transfer fluency $\diamond$ & 0 & - & - & 0 & - & $\bullet$ & O & - & $\bullet$ \\
\hline & Bite size adaptability & 0 & 0 & O & 0 & 0 & 0 & $x$ & $x$ & $x$ \\
\hline & Bite timing adaptability & - & - & - & ○ & $\bullet$ & $\bullet$ & 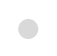 & 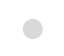 & $\odot$ \\
\hline & Bite sequence adaptability & 0 & 0 & - & $x$ & $x$ & $x$ & 0 & 0 & 0 \\
\hline \multirow{5}{*}{$\begin{array}{l}\text { User Empowerment and } \\
\text { Social Acceptance }\end{array}$} & Perceived usefulness * & O & O & - & O & O & - & 0 & O & - \\
\hline & Perceived ease of use & 0 & O & - & 0 & 0 & - & - & - & - \\
\hline & Attitude ${ }^{\star}$ & O & 0 & - & O & ○ & - & O & O & - \\
\hline & Intention to use * & - & - & - & - & - & - & 0 & 0 & - \\
\hline & Perceived enjoyment & O & O & - & - & - & - & - & - & - \\
\hline \multirow{3}{*}{ System Integration } & Onboard electronics & 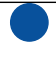 & 0 & O & & O & 8 & O & O & \\
\hline & Anomaly monitoring capability & $x$ & $x$ & $x$ & O & 0 & 0 & - & - & 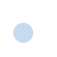 \\
\hline & Integration with other assistive devices & $x$ & $x$ & $x$ & $x$ & $x$ & $x$ & $x$ & $x$ & $x$ \\
\hline
\end{tabular}

\footnotetext{
${ }^{\dagger}$ Obi can scoop a variety of food items; the PR2 system can handle yogurt, cereal, cottage cheese, watermelon, fried rice, and mixed fruit; ADA can handle 16 different solid food items including salad leaves.

$\otimes$ Obi is portable but table-mounted and difficult to setup in a new environment; PR2 is mobile but it is difficult to move it everywhere a user goes.

* PR2 system can address a wider range of anomalies compared to ADA.

${ }^{\circ}$ Obi uses buttons (requires hand movement); PR2 uses GUI (requires some arm movement); ADA uses speech (requires voice).

$\diamond$ ADA can autonomously trigger feeding when the mouth is open.

$¥$ These systems cannot adapt autonomously. They can be triggered by a user and thus, can adapt to the pace and portion as needed. ADA has the capability to detect and select a particular food item on a plate autonomously, and can thus follow a user-specified bite sequence.

* For ADA, evaluations were done using a robot video.
}

\section{Evaluation}

Based on the evaluation indicators developed in the metrics subsection, we performed an evaluation of the three robotic systems: Obi, PR2, and ADA. Detailed results are given in Table 1 and Fig. 5. Note, not all the measures were available for these robotic systems publicly and thus, for the unavailable information, we put a '-' instead on the table. Note, though
Obi is commercially available, some of the known barriers to its user acceptance as reported by one of its distributors are due to minimal autonomy. For example, the most frequent problem is that people 'bite' the spoon hard which makes it fall off and then the user can not reattach it by themselves due to their impairments. 


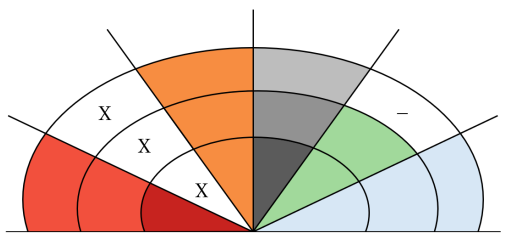

(a) Obi [7]

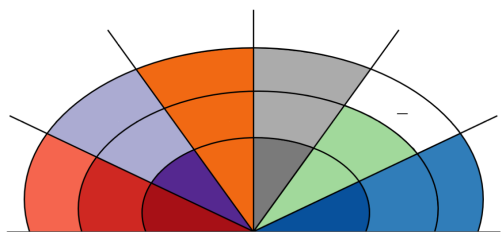

(b) PR2 [62]

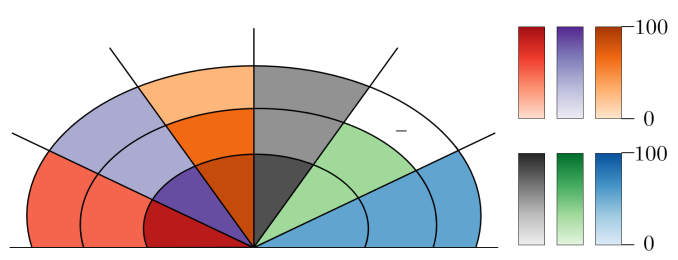

(c) ADA [36]

Figure 5: Summary comparison: we used the average for each level from Table 1. Darker colors imply higher ratings.

\section{DISCUSSION}

Evaluating RAF systems from a social-ecological approach presents a number of limitations. First, it is not obvious how to facilitate data collection and assess the reliability of data within the four levels of influence (in particular the larger scale ones). An exciting research challenge will be to understand the heterogeneous technological competencies and technology adoption rates among the various levels. As a sensing technology, RAF systems may make detailed quantitative data available for questions for which only qualitative data has traditionally been available, and data analysis could displace statistical approximations in examining certain questions at the macro-levels. Exploring the possibilities for evaluating RAF systems as multi-leveled through the SEM lens is the first step towards developing a universal reporting system that will provide comparable data related to each level of the model. The next challenge will be developing ways to synthesize multi-level information to draw conclusions that are broad enough for generalization yet specific enough to be useful to policy and community stakeholders. In addition, using this evaluation framework, we have identified 3 research milestones that our system, ADA, needs to achieve in the future: a) User Empowerment and Social Acceptance: Testing with end-users, b) Technology Robustness: Anomaly detection and correction, and c) Technical Function: "Range of food items". These were chosen not only based on the scores and where we lack compared to other systems based on these scores but these were also validated from user, caregiver and/or expert responses through our previous contextual inquiry.

\section{ACKNOWLEDGMENTS}

This work was funded by the National Institute of Health R01 (\#R01EB019335), National Science Foundation CPS (\#1544797), National Science Foundation NRI (\#1637748), the Office of Naval Research, the RCTA, Amazon, and Honda. The Taskar Center supported Anat Caspi's engagement.

\section{REFERENCES}

[1] 2018. Agis Living. (2018). http://www.agis .com,[Online; Retrieved on 14th May, 2018].

[2] 2018. JACO Robotic Arm. (2018). https://www.kinovarobotics.com/en/products/ robotic-arm-series, [Online; Retrieved on 27th August, 2018].

[3] 2018a. Meal Buddy. (2018). https://www. performancehealth. com/meal-buddy-system,[Online; Retrieved on 25th January, 2018].
[4] 2018b. Meal-Mate. (2018). https://www .made2aid.co.uk/productprofile?productId= 8\&company=RBF\%20Heal thcare\&product=Meal-Mate,[Online; Retrieved on 25th January, 2018].

[5] 2018c. The Mealtime Partner Dining System. (2018). http://mealtimepartners.com/,[Online; Retrieved on 15th February, 2018].

[6] 2018. My Spoon. (2018). https: //www. secom.co.jp/english/myspoon/food.html,[Online; Retrieved on 25th January, 2018].

[7] 2018. Obi. (2018). https://meetobi.com/,[Online; Retrieved on 25th January, 2018].

[8] 2018. ROVI Wheelchair. (2018). http://www.rovimobility.com/,[Online; Retrieved on 27th August, 2018].

[9] 2018. Winsford Feeder. (2018). https://www . youtube . com/watch?v=KZRF j1UZl-c,[Online; Retrieved on 15th February, 2018].

[10] 2019. Beeson Automaddak Feeder. (2019). https://abledata.acl.gov/product/ beeson-automaddak-feeder-model-h74501,[Online; Retrieved on 18th April, 2019].

[11] 2019. Bestic. (2019). https://www . camanio.com/us/products/bestic/,[Online; Retrieved on 18th April, 2019].

[12] 2019. Jamie. (2019). https: //www. focalmeditech.nl/en/content/jamie,[Online; Retrieved on 18th April, 2019].

[13] 2019. Liftware handles. (2019). https://www.liftware.com/,[Online; Retrieved on 18th April, 2019].

[14] 2019. Neater Eater Robot. (2019). http://www.neater. co.uk/neater-eater-2-2/,[Online; Retrieved on 18th April, 2019].

[15] 2019. Nelson. (2019). https: //www. focalmeditech.nl/en/content/nelson,[Online; Retrieved on 18th April, 2019].

[16] Redwan M Alqasemi, Edward J McCaffrey, Kevin D Edwards, and Rajiv V Dubey. 2005.

Wheelchair-mounted robotic arms: Analysis, evaluation and development. In Proceedings, 2005 IEEE/ASME International Conference on Advanced Intelligent Mechatronics. IEEE, 1164-1169. 
[17] Anders Bäckström, Astrid Norberg, and B O Norberg. 1987. Feeding difficulties in long-stay patients at nursing homes. Caregiver turnover and caregivers' assessments of duration and difficulty of assisted feeding and amount of food received by the patient. International journal of nursing studies 241 (1987), 69-76.

[18] Tapomayukh Bhattacharjee, Gilwoo Lee, Hanjun Song, and Siddhartha S Srinivasa. 2019. Towards Robotic Feeding: Role of Haptics in Fork-based Food Manipulation. IEEE Robotics and Automation Letters (2019).

[19] Zeungnam Bien, Myung-Jin Chung, Pyung-Hun Chang, Dong-Soo Kwon, Dae-Jin Kim, Jeong-Su Han, Jae-Hean Kim, Do-Hyung Kim, Hyung-Soon Park, Sang-Hoon Kang, and others. 2004. Integration of a rehabilitation robotic system (KARES II) with human-friendly man-machine interaction units. Autonomous robots 16, 2 (2004), 165-191.

[20] Jonathan Bohren, Radu Bogdan Rusu, E Gil Jones, Eitan Marder-Eppstein, Caroline Pantofaru, Melonee Wise, Lorenz Mösenlechner, Wim Meeussen, and Stefan Holzer. 2011. Towards autonomous robotic butlers: Lessons learned with the PR2. In 2011 IEEE International Conference on Robotics and Automation. IEEE, 5568-5575.

[21] Matthew W Brault. 2012. Americans with disabilities: 2010. Current population reports 7 (2012), 70-131.

[22] Joost Broekens, Marcel Heerink, Henk Rosendal, and others. 2009. Assistive social robots in elderly care: a review. Gerontechnology 8, 2 (2009), 94-103.

[23] Steven W Brose, Douglas J Weber, Ben A Salatin, Garret G Grindle, Hongwu Wang, Juan J Vazquez, and Rory A Cooper. 2010. The role of assistive robotics in the lives of persons with disability. American Journal of Physical Medicine \& Rehabilitation 89, 6 (2010), 509-521.

[24] Gerard Canal, Guillem Alenyà, and Carme Torras. 2016. Personalization framework for adaptive robotic feeding assistance. In International Conference on Social Robotics. Springer, 22-31.

[25] Alexandre Candeias, Travers Rhodes, Manuel Marques, Manuela Veloso, and others. 2018. Vision Augmented Robot Feeding. In Proceedings of the European Conference on Computer Vision (ECCV). 0-0.

[26] Anat Caspi, Mark Hallenbeck, and Shannon Tyman. 2019. USDOT ATTRI Evaluation Framework Briefing. (2019). https://arxiv.org/submit/2776975/view.

[27] Ke Chen and Alan Hoi Shou Chan. 2014. Gerontechnology acceptance by elderly Hong Kong Chinese: a senior technology acceptance model (STAM). Ergonomics 57, 5 (2014), 635-652.

[28] Graeme Cooke and Rick Muir. 2012. The relational state: How recognising the importance of human relationships could revolutionise the role of the state. IPPR.

[29] Fred D Davis. 1989. Perceived usefulness, perceived ease of use, and user acceptance of information technology. MIS quarterly (1989), 319-340.

[30] Fred D Davis, Richard P Bagozzi, and Paul R Warshaw. 1989. User acceptance of computer technology: a comparison of two theoretical models. Management science 35, 8 (1989), 982-1003. DOI : http://dx.doi.org/10.1287/mnsc. 35.8.982

[31] Hkan Eftring and Kerstin Boschian. 1999. Technical results from manus user trials. In Proc. ICORR, Vol. 99. 136-141.

[32] M Evans. 1991. Magpie: It's development and evaluation. Technical Report. Technical report.

[33] Neta Ezer, Arthur D Fisk, and Wendy A Rogers. 2009. Attitudinal and intentional acceptance of domestic robots by younger and older adults. In Universal Access in Human-Computer Interaction. Intelligent and Ubiquitous Interaction Environments. Springer, 39-48. DOI : http://dx.doi .org/10 .1007/978-3-642-02710-9_5

[34] National Alliance for Caregiving. 2005. Caregiving in the US. AARP; Bethesda, MD: The National Alliance for Caregiving.

[35] Christopher Frauenberger. 2015. Disability and technology: A critical realist perspective. In Proceedings of the 17th International ACM SIGACCESS Conference on Computers \& Accessibility. ACM, 89-96.

[36] Daniel Gallenberger, Tapomayukh Bhattacharjee, Youngsun Kim, and Siddhartha S Srinivasa. 2019. Transfer depends on Acquisition: Analyzing Manipulation Strategies for Robotic Feeding. In ACM/IEEE International Conference on Human-Robot Interaction (HRI).

[37] Phillip M Grice, Yash Chitalia, Megan Rich, Henry Clever, Henry Evans, Jane Evans, and Charles C Kemp. 2016. Autobed: A Web-Controlled Robotic Bed. Technical Report. Georgia Institute of Technology.

[38] Marcel Heerink, Ben Kröse, Vanessa Evers, and Bob Wielinga. 2010. Assessing acceptance of assistive social agent technology by older adults: the almere model. International journal of social robotics 2, 4 (2010), 361-375.

[39] Marcel Heerink, Ben Kröse, Bob Wielinga, and Vanessa Evers. 2008. Enjoyment intention to use and actual use of a conversational robot by elderly people. In Proceedings of the 3rd ACM/IEEE international conference on Human robot interaction. ACM, 113-120. DOI : http://dx. doi .org/10.1145/1349822.1349838

[40] Laura V Herlant. 2016. Algorithms, Implementation, and Studies on Eating with a Shared Control Robot Arm. Ph.D. Dissertation. 
[41] Hiroki Higa, Kei Kurisu, and Hideyuki Uehara. 2014. A vision-based assistive robotic arm for people with severe disabilities. Transactions on Machine Learning and Artificial Intelligence 2, 4 (2014), 12-23.

[42] M Hillman and A Gammie. 1994. The Bath institute of medical engineering assistive robot. In Proc. ICORR, Vol. 94. 211-212.

[43] Alberto Jardón, Concepción A Monje, and Carlos Balaguer. 2012. Functional evaluation of ASIBOT: A new approach on portable robotic system for disabled people. Applied Bionics and Biomechanics 9, 1 (2012), 85-97.

[44] S. Javdani, H. Admoni, S. Pellegrinelli, S.S. Srinivasa, and J.A. Bagnell. 2018. Shared Autonomy via Hindsight Optimization for Teleoperation and Teaming. The International Journal of Robotics Research (2018). (To appear).

[45] Vijay Kumar, Tariq Rahman, and Venkat Krovi. 1997. Assistive devices for people with motor disabilities. Wiley Encyclopedia of Electrical and Electronics Engineering (1997).

[46] I. F. Lin and H. S. Wu. 2011. Does informal care attenuate the cycle of ADL/IADL disability and depressive symptoms in late life. The Journals of Gerontology Series B: Psychological Sciences and Social Sciences 66, 5 (2011), 585594.

[47] P Lopes, R Lavoie, R Faldu, N Aquino, J Barron, M Kante, and B Magfory. 2012. Icraft-eye-controlled robotic feeding arm technology. Tech. Rep. (2012).

[48] Dennis Maciuszek, Johan Aberg, and Nahid Shahmehri. What Help Do Older People Need? Constructing a Functional Design Space of Electronic Assistive Technology Applications. (????).

[49] Veronique Maheu, Philippe S Archambault, Julie Frappier, and François Routhier. 2011. Evaluation of the JACO robotic arm: Clinico-economic study for powered wheelchair users with upper-extremity disabilities. In Rehabilitation Robotics (ICORR), 2011 IEEE International Conference on. IEEE, 1-5.

[50] Jennifer Mankoff, Gillian R Hayes, and Devva Kasnitz. 2010. Disability studies as a source of critical inquiry for the field of assistive technology. In Proceedings of the 12th international ACM SIGACCESS conference on Computers and accessibility. ACM, 3-10.

[51] K. D. Marek, L. Popejoy, G. Petroski, D. Mehr, M. Rantz, and W.-C Lin. 2005. Clinical Outcomes of Aging in Place. 54, 3 (2005), 202-211.

[52] Bente Martinsen, Ingegerd Harder, and Fin Biering-Sorensen. 2008. The meaning of assisted feeding for people living with spinal cord injury: a phenomenological study. Journal of advanced nursing 625 (2008), 533-40.

[53] Derek McColl and Goldie Nejat. 2013. Meal-time with a socially assistive robot and older adults at a long-term care facility. Journal of Human-Robot Interaction 2, 1 (2013), 152-171.

[54] Michelle E Mlinac and Michelle C Feng. 2016. Assessment of Activities of Daily Living, Self-Care, and Independence. Archives of Clinical Neuropsychology 31, 6 (2016), 506-516.

[55] Rick Muir and Imogen Parker. 2014. Many to many: How the relational state will transform public services. IPPR.

[56] Maia Naftali and Leah Findlater. Accessibility in Context: Understanding the Truly Mobile Experience of Smartphone Users with Motor Impairments. (????).

[57] Isira Naotunna, Chamika Janith Perera, Chameera Sandaruwan, RARC Gopura, and Thilina Dulantha Lalitharatne. 2015. Meal assistance robots: A review on current status, challenges and future directions. In 2015 IEEE/SICE International Symposium on System Integration (SII). IEEE, 211-216.

[58] Eiichi Ohara, Ken'ichi Yano, Satoshi Horihata, Takaaki Aoki, and Yutaka Nishimoto. 2009. Tremor suppression control of meal-assist robot with adaptive filter. In 2009 IEEE International Conference on Rehabilitation Robotics. IEEE, 498-503.

[59] Yutaro Ohshima, Yuichi Kobayashi, Toru Kaneko, Atsushi Yamashita, and Hajime Asama. 2013. Meal support system with spoon using laser range finder and manipulator. In 2013 IEEE Workshop on Robot Vision (WORV). IEEE, 82-87.

[60] D. Park, Z. Erickson, T. Bhattacharjee, and C. C. Kemp. 2016a. Multimodal execution monitoring for anomaly detection during robot manipulation. In 2016 IEEE International Conference on Robotics and Automation (ICRA). 407-414. DOI :

http://dx.doi.org/10.1109/ICRA.2016.7487160

[61] D. Park, H. Kim, Y. Hoshi, Z. Erickson, A. Kapusta, and C. C. Kemp. 2017. A multimodal execution monitor with anomaly classification for robot-assisted feeding. In 2017 IEEE/RSJ International Conference on Intelligent Robots and Systems (IROS). 5406-5413.

[62] Daehyung Park, You Keun Kim, Zackory M. Erickson, and Charles C. Kemp. 2016b. Towards Assistive Feeding with a General-Purpose Mobile Manipulator. CoRR abs/1605.07996 (2016). http://arxiv.org/abs/1605.07996

[63] Anupam Pathak, John A Redmond, Michael Allen, and Kelvin L Chou. 2014. A noninvasive handheld assistive device to accommodate essential tremor: a pilot study. Movement Disorders 29, 6 (2014), 838-842.

[64] Chamika Janith Perera, Thilina Dulantha Lalitharatne, and Kazuo Kiguchi. 2017. EEG-controlled meal assistance robot with camera-based automatic mouth position tracking and mouth open detection. In 2017 IEEE International Conference on Robotics and Automation (ICRA). IEEE, 1760-1765. 
[65] Chamika Janith Perera, Isira Naotunna, Chameera Sadaruwan, Ranathunga Arachchilage Ruwan Chandra Gopura, and Thilina Dulantha Lalitharatne. 2016. SSVEP based BMI for a meal assistance robot. In 2016 IEEE International Conference on Systems, Man, and Cybernetics (SMC). IEEE, 002295-002300.

[66] Lin Perry. 2008. Assisted feeding. Journal of advanced nursing 62, 5 (2008), 511-511.

[67] Travers Rhodes and Manuela Veloso. 2018. Robot-driven Trajectory Improvement for Feeding Tasks. In 2018 IEEE/RSJ International Conference on Intelligent Robots and Systems (IROS). IEEE, 2991-2996.

[68] WC Richardson, DM Berwick, J Bisgard, L Bristow, C Buck, and C Cassel. 2001. Institute of Medicine. Crossing the Quality Chasm: A New Health System for the 21st Century. (2001).

[69] Sebastian Schröer, Ingo Killmann, Barbara Frank, Martin Völker, Lukas Fiederer, Tonio Ball, and Wolfram Burgard. 2015. An autonomous robotic assistant for drinking. In 2015 IEEE International Conference on Robotics and Automation (ICRA). IEEE, 6482-6487.

[70] Cory-Ann Smarr, Tracy L Mitzner, Jenay M Beer, Akanksha Prakash, Tiffany L Chen, Charles C Kemp, and Wendy A Rogers. 2014. Domestic robots for older adults: Attitudes, preferences, and potential. International Journal of Social Robotics 6, 2 (2014), 229-247. DOI :

http://dx.doi.org/10.1007/s12369-013-0220-0

[71] Won-Kyung Song and Jongbae Kim. 2012. Novel assistive robot for self-feeding. In Robotic Systems-Applications, Control and Programming. IntechOpen.

[72] Rebecca Q Stafford, Bruce A MacDonald, and Elizabeth Broadbent. 2012. Identifying specific reasons behind unmet needs may inform more specific eldercare robot design. In International Conference on Social Robotics. Springer, 148-157.

[73] Sarit Felicia Anais Szpiro, Shafeka Hashash, Yuhang Zhao, and Shiri Azenkot. 2016. How People with Low Vision Access Computing Devices: Understanding Challenges and Opportunities. In Proceedings of the 18th International ACM SIGACCESS Conference on Computers and Accessibility (ASSETS '16). ACM, New York, NY, USA, 171-180.

[74] Yoshihiko Takahashi and Shinichiro Suzukawa. 2006. Easy human interface for severely handicapped persons and application for eating assist robot. In 2006 IEEE International Conference on Mechatronics. IEEE, 225-229.

[75] Kanya Tanaka, Shenglin Mu, and Shota Nakashima. 2014. Meal-assistance robot using ultrasonic motor with eye interface. International Journal of Automation Technology 8, 2 (2014), 186-192.

[76] Noriyuki Tejima. 1996. Evaluation of rehabilitation robots for eating. In Robot and Human Communication, 1996., 5th IEEE International Workshop on. IEEE, 118-120.

[77] Mike Topping, Helmut Heck, Gunnar Bolmsjo, and David Weightman. 1998. The development of RAIL (robotic aid to independent living). In Proceedings of the third TIDE Congress.

[78] Michael J Topping and Jane K Smith. 1999. The development of handy 1 . A robotic system to assist the severely disabled. Technology and Disability 10, 2 (1999), 95-105.

[79] Mitra Unosson, A C Ek, P. Bjurulf, H H von Schenck, and J O Larsson. 1994. Feeding dependence and nutritional status after acute stroke. Stroke 252 (1994), 366-71.

[80] Loek A Van der Heide, Bob Van Ninhuijs, Arjen Bergsma, Gert Jan Gelderblom, Dick J Van Der Pijl, and Luc P De Witte. 2014. An overview and categorization of dynamic arm supports for people with decreased arm function. Prosthetics and orthotics international 38, 4 (2014), 287-302.

[81] Hans Van der Heijden. 2004. User acceptance of hedonic information systems. MIS quarterly (2004), 695-704.

[82] Viswanath Venkatesh and Michael G Morris. 2000. Why don't men ever stop to ask for directions? Gender, social influence, and their role in technology acceptance and usage behavior. MIS quarterly (2000), 115-139.

[83] Viswanath Venkatesh, Michael G Morris, Gordon B Davis, and Fred D Davis. 2003. User acceptance of information technology: Toward a unified view. MIS quarterly (2003), 425-478.

[84] Viswanath Venkatesh, James YL Thong, and Xin Xu. 2012. Consumer acceptance and use of information technology: extending the unified theory of acceptance and use of technology. (2012).

[85] Maria Vila Abad, Gerard Canal Camprodon, and Guillem Alenyà Ribas. 2018. Towards safety in physically assistive robots: eating assistance. In Proceedings of the 2018 IROS Workshop on Robots for Assisted Living. 1-4.

[86] Akira Yamazaki and Ryosuke Masuda. 2012a. Autonomous foods handling by chopsticks for meal assistant robot. In ROBOTIK 2012; 7th German Conference on Robotics. VDE, 1-6.

[87] Akira Yamazaki and Ryosuke Masuda. 2012b. Various foods handling movement of chopstick-equipped meal assistant robot and there evaluation. In International Conference on Social Robotics. Springer, 158-167. 